\title{
Perceiving space through sound: mapping human movements into MIDI
}

\author{
Bernardo Breve, Stefano Cirillo, Domenico Desiato \\ Department of Computer Science \\ University of Salerno \\ 84084 Fisciano (SA), Italy \\ \{bbreve,scirillo,ddesiato\}@unisa.it \\ Mariano Cuofano \\ MRes Architecture \\ Royal College of Art \\ South Kensington, London (UK) \\ mariano.cuofano@alumni.rca.ac.uk
}

\begin{abstract}
Gestural expressiveness plays a fundamental role in the interaction with people, environments, animals, things, and so on. Thus, several emerging application domains would exploit the interpretation of movements to support their critical designing processes. To this end, new forms to express the people's perceptions could help their interpretation, like in the case of music. This paper presents a novel algorithm for mapping human movements into MIDI music. The algorithm has been implemented in a system that integrates a module for real-time tracking of movements through a sample-based synthesizer using different types of filters to modulate frequencies. The system has been evaluated through a user study, in which several users have participated to a room experience, yielding significant results about their perceptions with respect to the environment they were immersed.
\end{abstract}

Index terms - Movements Tracking, MIDI Sound, Synthesizer Sounds

\section{Introduction}

Gestures, movements, and body languages represent a common way through which it is possible to mark verbal communication. Indeed, they emphasise the language by adding significant characteristics useful for communication purposes. More specifically, it is possible to capture details that allow to associate a particular gesture with an emotion. In fact, by analysing the interaction between two persons, it is possible to understand the feelings produced during their communication through their gestures. In the state of the art, it is possible to find different tracking techniques for detecting human movements [30], and techniques to represent their semantics [1]. They have been mainly applied to scenarios in which it is necessary to support humans in the real-time interpretation of critical situations, such as in the context of video surveillance [8] and emergency management [28]. In the social context, an important role is played by the music. It is possible to define music as an art manifestation, since it consists of creating and producing sounds that are pleasant to the human ear. The music, in most cases, leads emotions in individuals who are providing and listening to it. Thus, also in this case, it is possible to associate a sentiment to a specific organisation of sounds. In fact, most of the time, people use music to regulate their emotions, this means that each individual through the listening of particular shades of sound modifies his/her emotional state or, more simply, a sound can create emotions such as happiness, sadness, gladness, and so on [11].

In this paper, we exploit the combination of human movements and sound synthesis techniques to associate sounds to movements in the space. More specifically, we propose a novel algorithm and a system for recognising human movements, and translating them into MIDI music, with a sample-based synthesizer, which uses different types of filters to modulate frequencies. The single frequency is used to associate a specific set of sounds to each movement captured in real-time. In particular, in order to quickly map spatial coordinates of people in sound, we defined a layer-based module, which is able to uniquely identify MIDI notes, and to manage their changes according to people's movements. 
The paper is organised as follows. In Section 2, we describe recent works concerning movements tracking techniques and their application into several fields. In Section 3 , we introduce some preliminary concepts useful to understand our proposal. In Section 4, we explain our methodology, whereas its validation is discussed in Section 5. Finally, conclusions and future research directions are provided in Section 6.

\section{Related work}

In this section, we describe approaches and methodologies defined in the literature concerning the mapping of gesture recognition $[3,12,13]$. In particular, we focus on different application domains in which the recognition of gestures plays an important role [21].

In the music field, interesting pioneering work is described in [20]. Authors present Conductor's Jacket, a wearable device interpreting physiological and gestural stimuli in order to apply them in a musical context. In fact, it uses sixteen sensors communicating with a musical software by collecting data over different reliable channels, also offering mcal-time graphical feedbacks to control the mapped gestures. Instead, in [27] authors present MATRIX (Multipurpose Array of Tactile Rods for Interactive eXpression), a musical interface for music amateurs and professionals. It permits the usage of hands to control music by exploiting a 3-dimensional interface allowing the manipulation of traditional musical instruments in conjunction with it. The MATRIX interface manipulates the parameters of a synthesis engine or effect algorithm in real-time, in response to the performer's expressive gestures. In [10], authors illustrate a real-time musical conducting gesture recognition system that supports music players in enhancing their performance. They used a single-depth camera to capture image inputs and to establish a real-time gesture recognition system. In the data mining field, one of the most relevant works is [26] in which the authors propose an innovative framework for progressive mining and querying of motion data, by also exploiting information extracted from data relationships [6].

Another application field concerns the application of gesture recognition applied to video surveillance. In [25], authors present a video surveillance framework for realtime multi-person tracking. It uses an adaptive background subtraction in order to identify foreground regions for catching users' movements. In [29], authors present an online approach to simultaneously detect $2 \mathrm{D}$ poses of multiple people in a video sequence. They exploit Part Affinity Field (PAF) representations designed for static images, and they propose an architecture that can encode Spatio-Temporal Affinity Fields (STAF) across a video sequence. In [32], authors present a novel multi-person tracking system for crowd counting and normal/abnormal events detection in indoor/outdoor surveillance environments. They use two challenging video surveillance datasets, such as PETS2009 and UMN crowd analysis datasets, to demonstrate the effectiveness of their proposed system, which achieved $88.7 \%$ and $95.5 \%$ of accuracy and detection rate, respectively. In [4] authors propose an algorithm for multi-person tracking in indoor surveillance systems based on a tracking-bydetection approach. They use Convolutional Neural Networks (CNNs) for detecting and tracking people. They also perform several experiments by tracking people in rapidpanic scenarios, achieving good performances in terms of classification accuracy. In [24], authors define a lightweight tracking algorithm named Kerman (Kernelized Kalman filter), which is a decision tree based hybrid Kernelized Correlation Filter (KCF) algorithm for human object tracking.

Finally, in the medical field, gesture recognition is applied to monitor diseases. Authors in [22] present an innovative approach to preterm-infants' limb pose estimation. They exploit spatio-temporal information to determine and track limb joint position from depth videos with high reliability. Instead, in [34], authors illustrate Ultigesture wristband, a hardware/software platform for gesture recognition and remote control. Ultigesture wristband offers full open API for third party research and application development.

\section{Preliminaries}

In this section, we provide preliminary notions to allow for a better understanding of the concepts underlying the proposed system.

The Musical Instrument Digital Interface (MIDI) is a standard de facto for enabling communication among digital musical instruments and processors of digital music, such as personal computers and sequencers [23]. A MIDI message carries data concerning the peculiarity of a certain sound, such as the vibrato, the tremolo, and so on. However, a MIDI file does not contain any actual waveform generated by the notes of a played musical instrument. Instead, it is a collection of data informing on how a type of sound can be simulated by the digital music processor, which is then responsible for playing the sound by retrieving the representation of the simulated music instrument assigned to those notes from its memory [14].

More specifically, a MIDI message is transmitted over 16 different channels in groups of 8 bits, each of which can be of two types called status byte and data byte, respectively. The latter defines the value associated with the message, whereas the former is used to specify the type of message sent. The bytes are distinguished through the first bit, that is, a status byte begins with the bit 1 , whereas a data byte begins with the bit 0 . A MIDI message is usually composed of a status byte, followed by one or two data bytes, and can belong to one of the two following categories: channel 




Figure 1: An example of MIDI message.

message or system message. Channel messages are sent to single channels and contain information about the musical performance; system messages are aimed at the MIDI system responsible for coordinating the succession of sounds.

Figure 1 shows an example of a MIDI message. In particular, it shows a channel message, composed of one status byte, which is mandatory for every type of message that has been sent and two data byte. The status byte contains information about the operation to be performed and the channel involved with that particular operation, which in this case of Figure 1 is "play a note on the third channel". The following two data bytes contain information about the note to be played and the velocity to be applied. The velocity is a value though which it is possible to emulate the amount of force exerted on the key. Alternatively, it can also describe the width of the output or the tone of the sound.

MIDI control changes, also known as or associated with MIDI Controllers or Control Changes, are MIDI messages conveying positional information related to performance control devices such as wheels, sliders, pedals, switches, and other control-oriented devices [31]. This type of information can be used to control a variety of functions, such as vibrato depth, brightness, and many other parameters. MIDI controller change messages are 128, and each of these has a control number and a control value parameter.

MIDI notes and control change are used in our system to map the spatial coordinates to sounds and to give a different effect to each sound. Details about the proposed system and on how it uses MIDI messages are provided in the next section.

\section{A system for mapping human movements into sounds}

We propose a new system named PIANO (maPpIng humAn movements iNto sOunds), which recognizes the movements of people in space and translates them into sounds.

PIANO is a modular system offering standalone modules, designed to be fast and easy to use, relying on non expensive hardware devices, available on the market. More specifically, PIANO consists of several modules, the first of which is a digital tracker relying on an infrared camera to distinguish the participants' bodies and associate them to a virtual figure. When a person enters the room, movements are mapped into sounds. These are read by Cubase, a sequencer located on a platform and emitted as sound.

\subsection{The Object/Human Detection Algorithm}

Tracking movements of a person in a live video is not always a simple operation. Thus, several problems must be considered, such as the variability. In fact, a position detection algorithm must be able to trace the object considering the enormous variations in the appearance and position of the objects. Therefore, there are several variables that a tracking algorithm must take into consideration: the point of view, the position, the lighting conditions, the quality or the occlusions of the images. Furthermore, the large number of successive frames that may belong to a live or recorded video can make tracking activities particularly difficult. A tracking system of a moving object must be able not to lose the position of the object in subsequent frames. Thus, an algorithm must be able to consider the variation of all these variables and to perform real-time activities on any environment. These types of operations are even more complicated when needing to identify and/or track groups of objects/people.

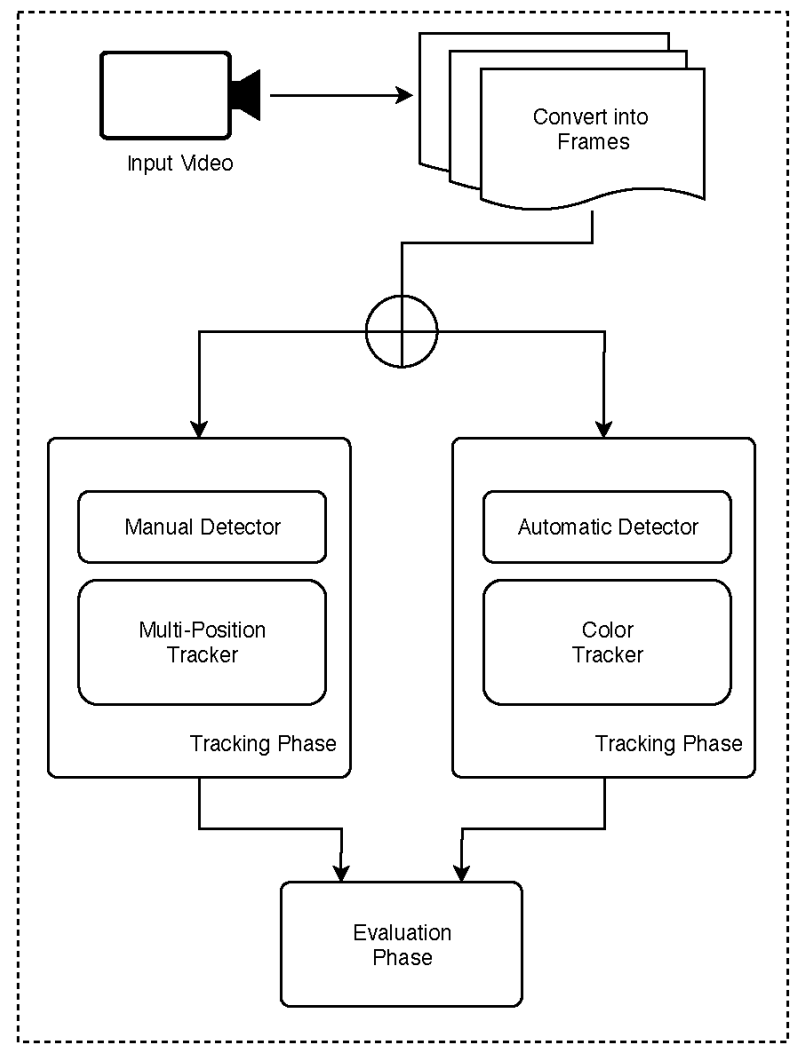

Figure 2: Flowchart of the object detection algorithm.

Within PIANO we designed a tracking module capable 
of using any camera for recognizing people. The proposed algorithm determines the position of one or many people. Starting from the video, it maps the position of each person in spatial coordinates.

Figure 2 shows the three phases of the PIANO tracking algorithm: detection of people, tracking of these subjects, and evaluation of the tracking results to describe semantic events and latent phenomena. In the first phase, the tracking algorithm reads the video as input and converts it to subsequent frames. In particular, the algorithm can work on static video sources or real-time input streams. This guarantees the high adaptability of PIANO to any type of situation and the repetition of itself several times, even after a live video recording. After selecting the input, PIANO allows choosing the tracking mode, i.e. manual or automatic.

Manual tracking enables a full control of the system and the arbitrary choice of whether or not to select a person to track. The user draws the selection rectangle of the objects or people s/he wants to monitor. In particular, each selection is a Region of Interest (ROI) to be tracked. Each selected $\mathrm{ROI}$ is associated with a person, and it will be independently recognised by the others. As shown in Figure 3(a), when a ROI is selected, the algorithm creates a virtual figure. Starting from this figure, the algorithm calculates the coordinates and defines the centre of the ROI. This is the reference point of the person's movement. Using different rectangles, it is possible to keep track of many people at the same time, so that PIANO can track separate music for each person. The algorithm can use different types of trackers: Boosting [15], Multiple Instance Learning (MIL) [2], Kernelized Correlation Filters (KCF) [16], TLD[18], Median-Flow [17], Minimum Output Sum of Squared Error (MOSSE) [5], and Discriminative Correlation Filter with Channel and Spatial Reliability (CSRT) [19]. Support for different types of trackers enables the algorithm to adapt itself to all tracking situations, regardless of hardware characteristics.

Automatic tracking has been created to track people in a dark room using an infrared camera. Unlike manual tracking, there is no need to select people or objects to track. The proposed tracker receives an input dictionary of colours, and after selecting the input, the individual frames are analysed. Each frame is resized, blurred, and converted to the HSV (Hue, Saturation, Value) colour space. Next, for each colour defined in the dictionary, the tracker checks objects in each frame. Then, it constructs a mask for the colour from the dictionary, and performs a series of implicit dilations and erosions in order to remove any small blobs left in the mask. Successively, it selects one of the colours in the dictionary and defines the contours of the figure, showing it within the frames. The shapes are defined as circles, and the centre is the reference point for the person's movements. Since this type of tracker has been created for shooting in the dark, two-colour scales have been defined in the algo-
Algorithm 1 MAPP ING_SPACE_MIDI

INPUT: The coordinates $\mathrm{H}_{x}, \mathrm{H}_{y}$ of the person in the space extracted from the bounding box; The height $\mathrm{F}_{h}$ and the width $\mathrm{F}_{w}$ of the frame; The number of rectangles on $\mathrm{X}$-axis of the frame $\mathrm{S}_{w}$; The number of rectangles on Y-axis of the frame $S_{h}$

OUTPUT: The MIDI note value to be played

$$
\begin{aligned}
& \text { 1: } R_{\text {height }}^{1} \leftarrow \frac{F_{\text {height }}}{S_{h}} \\
& \text { 2: } R_{\text {width }}^{1} \leftarrow \frac{F_{\text {width }}}{S_{w}} \\
& \text { 3: } G_{x}^{1} \leftarrow \frac{H_{y}}{R_{\text {height }}^{1}} \\
& \text { 4: } G_{y}^{1} \leftarrow \frac{H_{x}}{R_{\text {width }}^{1}} \\
& \text { 5: } M I D I_{\text {note }} \leftarrow S_{h} * G_{x}^{1}+G_{y}^{1} \\
& \text { 6: return MIDI } I_{\text {note }}
\end{aligned}
$$

rithm, red and grey.

As shown in Figure 2, after the tracking phase, it is necessary to evaluate the output of each tracker. The evaluation phase defined in PIANO is described in the next section.

\subsection{Mapping space to sound}

PIANO uses two different functional systems: digital and analog. The analog system is based on a technology inspired by the "Theremin". It consists of an antenna capable of feeling the proximity of the electric voltage of the human body, and of translating it into an analog audio input, which is pre-amplified by an integrated circuit before issuing the sound through an active speaker. The three objects interacting with each other generate a magnetic field producing a white background noise. Bodies moving through this field alternate this with the magnetic status, and as a consequence, alter the white background noise and sound.

The digital system works on the actual relation of the bodies in the room, associating them to a sound only treated with their position in the room. In particular, we developed an algorithm to transform the movement of one or more people into sounds. This operation is particularly complex so that it is necessary to use a mapping methodology that is fast and precise. To this end, we have developed an approach to quickly map spatial coordinates to sound. After the video stream is read, the proposed methodology creates different virtual layers for each frame: the first layer defines the midi note that must be played, whereas the second layer represents the control change for each note. Figure 3 shows how the frame is divided. In particular, Figure 3(a) shows a representation of a frame from a video stream. We divided each frame into 128 different equal parts, corresponding to 


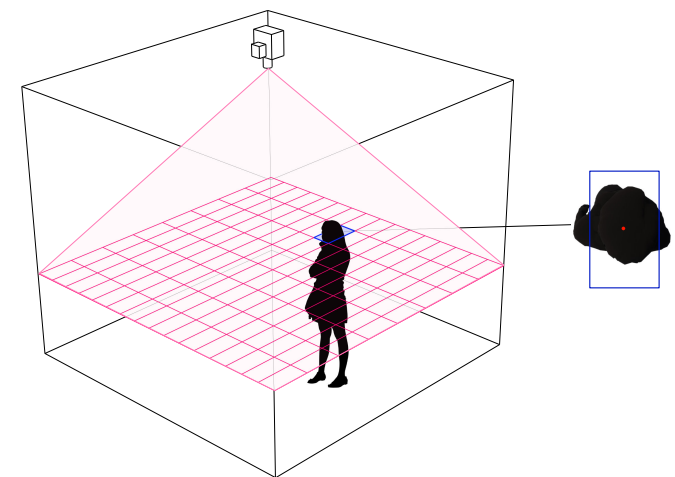

(a)

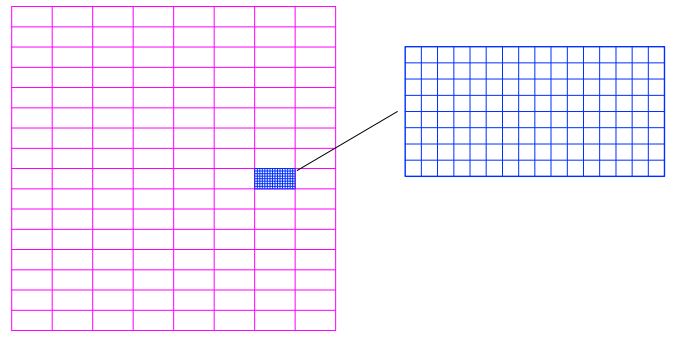

(b)

Figure 3: Virtual grids in the environment.

the number of existing midi notes. To do this, PIANO automatically calculates the size of each section.

Formally, let $\mathrm{F}$ be the frame extracted from the video, $\mathrm{F}_{\text {width }}$ and $\mathrm{F}_{\text {height }}$ the height and the width of $\mathrm{F}$, respectively, both expressed in pixel, $\mathrm{S}_{h}$ and $\mathrm{S}_{w}$ the maximum number of rectangles on $\mathrm{F}_{\text {width }}$ and $\mathrm{F}_{\text {height }}$, i.e. $\mathrm{S}_{w}=16$ and $S_{h}=8$. Then, starting from the centre of the bounding box identified on the frame at the position $\left(\mathrm{H}_{x}, \mathrm{H}_{y}\right)$, it is necessary to use the following formulas to define the midi note to play:

$$
\begin{aligned}
G_{x}^{1} & =\frac{H_{x} \cdot S_{w}}{F_{w i d t h}} \\
G_{y}^{1} & =\frac{H_{y} \cdot S_{h}}{F_{\text {height }}}
\end{aligned}
$$

Moreover, Figure 3(b) shows the structure of the second layer. In particular, each rectangle of the first layer is in turn divided into 128 rectangles of equal size, which represent the control change. When the centre of the bounding box plays a midi note in the first layer, the control change is simultaneously set in the second layer. Starting from the formulas 1 and 2, we can define the type of control change when playing the midi note:

$$
\begin{aligned}
G_{x}^{2} & =\frac{\left(H_{x}-G_{x}^{1} \cdot \frac{F_{w i d t h}}{S_{w}}\right)}{2 \cdot S_{w}} \\
G_{y}^{2} & =\frac{\left(H_{y}-G_{y}^{1} \cdot \frac{F_{h e i g h t}}{S_{h}}\right)}{2 \cdot S_{h}}
\end{aligned}
$$

Using the formulas 1-4, we obtain a pair of coordinates $\left(G_{x}^{1}, G_{y}^{1}\right)$ and $\left(G_{x}^{2}, G_{y}^{2}\right)$ that allow us to identify the midi note and the control change in $\mathrm{F}$ to play.

Algorithm 1 shows the mapping function from spatial coordinates to MIDI notes. Given the size of each frame

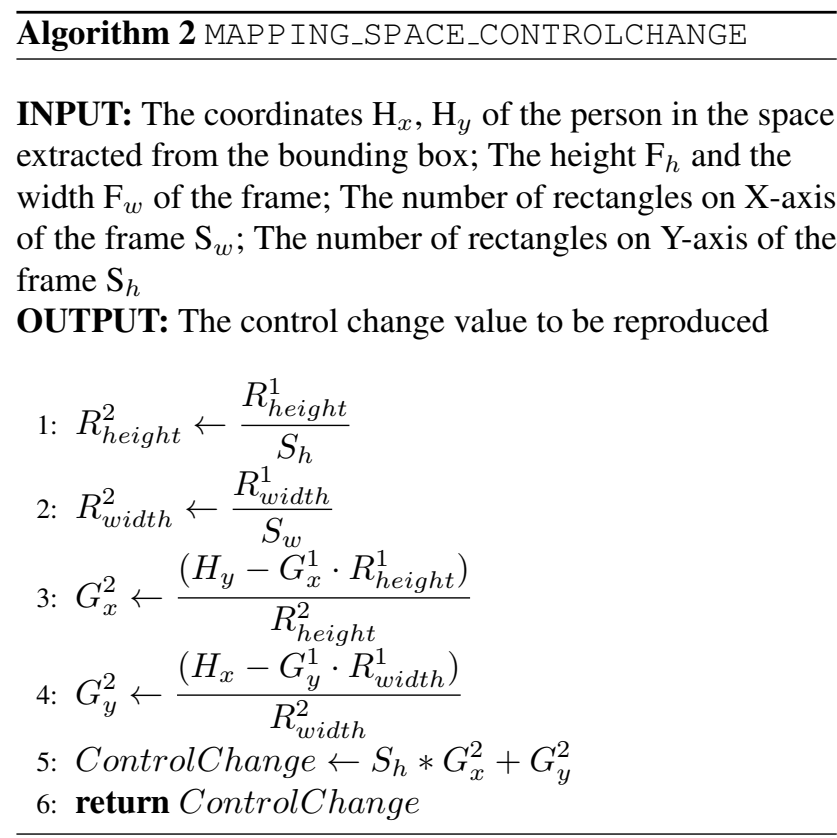

expressed in pixel, the number of rectangles in which the space has been divided, and the centre of the bounding box defined in the frame to track one person, the algorithm defines the height and the width of each rectangle in the first grid (lines 1-2), and then calculates the rectangle in which the person moves (lines 3-4). As said above, each rectangle on the grid corresponds to a different note, so that it is possible to define the MIDI note to be played by only considering the coordinates of the rectangle (line 5).

Algorithm 2 provides the control change value to be played. Similarly to Algorithm 1, it starts by considering the dimensions of each rectangle in the first grid, aiming to define the height and the width of the rectangles in the second grid (lines 1-2). As said above, the control change can take 128 possible values, each defined from a single rectan- 


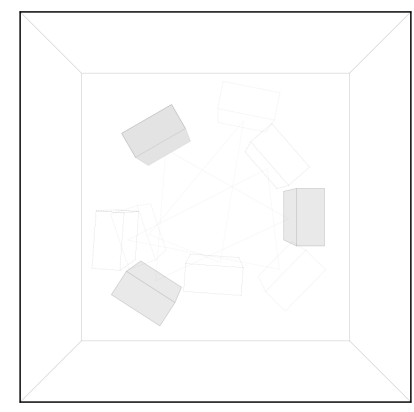

(a)

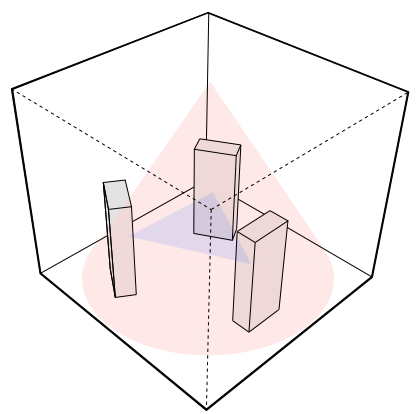

(b)

Figure 4: Interaction Room.

gle in the second grid. Thus, the algorithm calculates the control change to be reproduced by considering the size of the frame and the position of the people (lines 3-5).

\section{Interaction Room}

In order to present the case study, it is necessary to provide some details about the perception of space aiming to understand how the user perceives space through unconventional instruments such as music.

Starting from the definition provided in [33], we consider the space as a room. The term room is derived from the archaic English rum, which is similar to the German world Raum (space). This, in turn, refers to the Latin derivation rus - which can be translated as the act of making space. In this definition, the room is not described as a space associated to a specific role in the context where it is located, like for example, as a component of an apartment. Rather, room here is meant as a volume where phenomena take place. Our attempt is to understand the meaning of movements in the architecture domain and how to design the space based on them. The analysis starts by moving a critique to the existenzminimum, assuming it to be a course of predetermination paradigms of architectural design. The final outcomes of these models produce a monolithic city, able to grow and to allow for urban colonisation, triggering a wild world attitude to the gentrification of an urban environment. Starting from these ideas, we have been designed a specific environment called Interaction Room.

As spatial speculation, the Interaction Room represents a format of feedbacking architecture. It aims to offer an opportunity, a phenomenon, and a sound, to show how it is possible to generate sounds and emotions by using the space and the relative position each one takes within, coherently with the presence of other individuals.

Sounds are generated into two different modes. The objects mentioned before will have the analog system in- tegrated into them, which creates an unpredictable sound relation with each possible room layout. However, digital sounds are generated by using the methodology defined in Section 4.2. The intention is to merge these two solutions to have more a homogeneous and deep sound.

The experience aimed to learn how to be in a space together, generating a sound that depends on how people perceive the relation with space. Through artistic and digital approaches, we have given people the opportunity to discuss their space recognition experience.

\subsection{Experimental Design}

The Interaction Room has been designed as cubic structure with dimensions of 3 meters (Figure 4). Space is interrupted by the presence of three objects, which simulate limitations, edges, and walls, that the participants could use to describe their own layout (Figure 4(b)). In particular, these structures are parallelepipedic objects having dimensions of $1.7 \times 0.7$ x 0.3 meters, and a weighing less than $10 \mathrm{~kg}$.

PIANO has been executed on a computer with an Intel i9-9900k 3.6GHz 8-core CPU with $32 \mathrm{~GB}$ of RAM. The system interacted with an infrared camera with a resolution of 3 megapixels and an external sound card to reproduce the sounds. The latter has been connected to four 500-watt audio speakers located in the four corners of the room.

The evaluation session has been performed by involving people of different ages in supervised experience. In particular, people had different education levels, including high school diploma, master degree, and $\mathrm{PhD}$. Moreover, some of them were architects and surveyors with considerable past experience.

The overall experience was based on the production of sounds. The idea was to link the sounds produced by the movements of participants to a specific position in the room, so as to relate it to a specific sound frequency (a note). The experience consists of accessing into the room, which ap- 


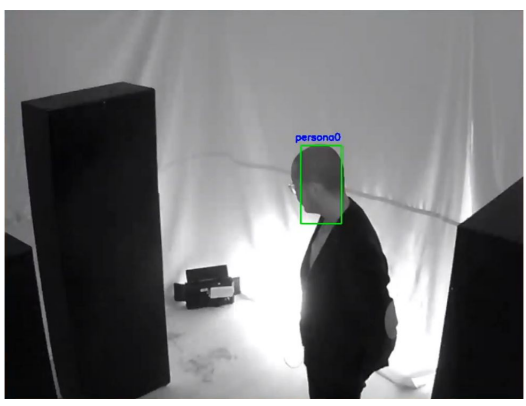

(a)

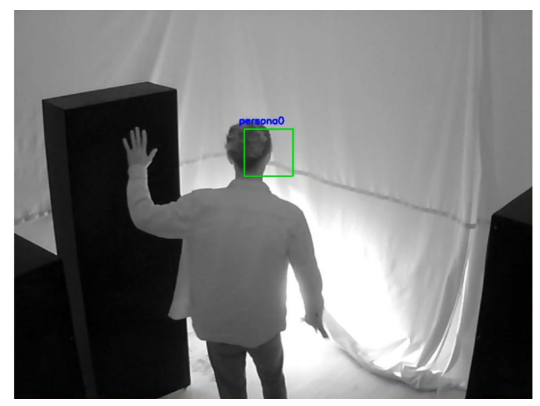

(b)

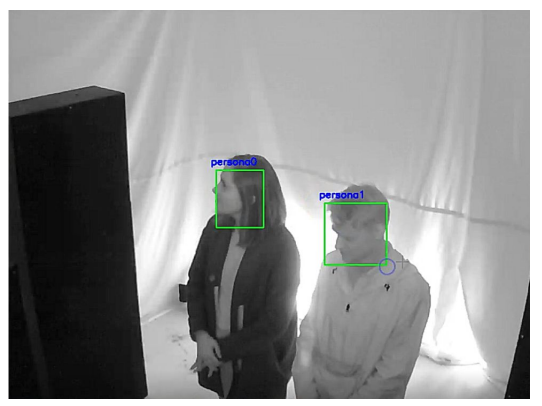

(c)

Figure 5: An example of multi-position tracker.

pears as dark volume. In this room, people were able to face other participants only as silhouettes, and the obscure environment did not enable them to distinguish proper figures and images. Shadows are generated by a tiny light located on the edges of the room.

The aim of this experiment is to achieve pure perceptive feedbacks from participants, without triggering an "overautomatisation" process in adapting to space.

\subsection{Experimental Results}

As the most frequent answer, people that took part in this experience were describing the capability of manipulating the space, and interacting with the sound. Describing direct feedback connected with participants' emotions, the room was able to manifest an interactive space, reproducing any movements in the form of sound.

From another point of view, it could be considered as a proper act of space production. It is clear how the environment perceived was not related to the tectonic of the room itself and the volume was not related to predictable habits. It is therefore clear how the act of making space was strictly related to a phenomenon [33].

Most of the participants claimed to have the impression of manipulation on the density of the room. This was possible because of the background white noise produced by the interference of the three objects. There was clearly a relation with the quantity of background noise and the quality of the space. Passing through this imaginary plan inhabitants were altering a system with their presence. What the objects were able to transmit is a clear form of feedback. It is revealing that through a perceptive operation it was possible to generate presence in relation to the environment. This elementary, primitive, generation of space represents the opportunity for creating architecture based on dynamic feelings and perceptions. This architecture is nor a tailored made space, neither the application of an efficient program, based on common functions and habits, rather it is strictly connected to the individual in that given moment. In other words, it could be assumed that this architecture was just a representation of those individuals in the form of space.

\section{Conclusion}

We proposed an algorithm and a system to recognize human movements in the space, aiming to translate them into sounds. The system has been used in the architectural domain, aiming to provide useful insights for gathering people's perceptions with respect to the surrounding space.

In the future, we would like to use the system into different application domains, such as video surveillance, and evaluate its performances. This type of system could be applied to define customized alarms in order to simply recognize the intents of possible malicious actions through the sounds. To this end, we would like to define a novel translation model in order to enable the possibility to map and distinguish high-level critical scenarios. Moreover, we would like to further extend our technique to exploit multimedia dependencies [9], since these can be nowadays automatically extracted from multimedia databases [7].

\section{References}

[1] P. L. Albacete, S. K. Chang, and G. Polese. Iconic language design for people with significant speech and multiple impairments, pages 12-32. Springer Berlin Heidelberg, Berlin, Heidelberg, 1998.

[2] B. Babenko, M.-H. Yang, and S. Belongie. Robust object tracking with online multiple instance learning. IEEE Transactions on Pattern Analysis and Machine Intelligence (TPAMI), 33(8):1619-1632, 2011.

[3] A. D. Bagdanov, A. Del Bimbo, L. Seidenari, and L. Usai. Real-time hand status recognition from rgb-d imagery. In Proc. of International Conference on Pattern Recognition (ICPR '12), page 2456-2459, 2012. 
[4] R. Bohush and I. Zakharava. Robust person tracking algorithm based on convolutional neural network for indoor video surveillance systems. In International Conference on Pattern Recognition and Information Processing, pages 289-300. Springer, 2019.

[5] D. S. Bolme, J. R. Beveridge, B. A. Draper, and Y. M. Lui. Visual object tracking using adaptive correlation filters. In 2010 IEEE computer society conference on computer vision and pattern recognition, pages 2544-2550. IEEE, 2010.

[6] L. Caruccio, S. Cirillo, V. Deufemia, and G. Polese. Incremental discovery of functional dependencies with a bitvector algorithm. In M. Mecella, G. Amato, and C. Gennaro, editors, Proceedings of the 27th Italian Symposium on Advanced Database Systems, volume 2400 of CEUR Workshop Proceedings. CEUR-WS.org, 2019.

[7] L. Caruccio, V. Deufemia, and G. Polese. Evolutionary mining of relaxed dependencies from big data collections. In Proceedings of the 7th International Conference on Web Intelligence, Mining and Semantics (WIMS), pages 5:1-5:10. ACM, 2017.

[8] L. Caruccio, G. Polese, G. Tortora, and D. Iannone. EDCAR: A knowledge representation framework to enhance automatic video surveillance. Expert Systems with Applications, 131:190-207, 2019.

[9] S. Chang, V. Deufemia, G. Polese, and M. Vacca. A normalization framework for multimedia databases. IEEE Transactions on Knowledge and Data Engineering, 19(12):16661679, 2007.

[10] F. Chin-Shyurng, S.-E. Lee, and M.-L. Wu. Real-time musical conducting gesture recognition based on a dynamic time warping classifier using a single-depth camera. Applied Sciences, 9(3):528, 2019.

[11] T. Cook, A. R. Roy, and K. M. Welker. Music as an emotion regulation strategy: An examination of genres of music and their roles in emotion regulation. Psychology of Music, 47(1):144-154, 2019.

[12] G. Costagliola, V. Deufemia, and M. Risi. A multi-layer parsing strategy for on-line recognition of hand-drawn diagrams. In Proc. of IEEE Symposium on Visual Languages and Human-Centric Computing (VL/HCC), pages 103-110, 2006.

[13] V. Deufemia, M. Risi, and G. Tortora. Sketched symbol recognition using latent-dynamic conditional random fields and distance-based clustering. Pattern Recognit., 47(3):1159-1171, 2014.

[14] M. Flam. Musical instrument digital interface with speech capability, 2001. US Patent 6,191,349.

[15] H. Grabner and H. Bischof. On-line boosting and vision. In Proc. IEEE Conference on Computer Vision and Pattern Recognition (CVPR'06), pages 260-267. IEEE, 2006.

[16] J. Henriques, R. Caseiro, P. Martins, and J. Batista. Highspeed tracking with kernelized correlation filters. IEEE transactions on pattern analysis and machine intelligence, 37(3):583-596, 2014.

[17] Z. Kalal, K. Mikolajczyk, and J. Matas. Forward-backward error: Automatic detection of tracking failures. In Proc. of International Conference on Pattern Recognition, pages 2756-2759, 2010.
[18] Z. Kalal, K. Mikolajczyk, and J. Matas. Tracking-learningdetection. IEEE Transactions on pattern analysis and machine intelligence, 34(7):1409-1422, 2011.

[19] A. Lukezic, T. Vojir, L. Cehovin Zajc, J. Matas, and M. Kristan. Discriminative correlation filter tracker with channel and spatialreliability. International Journal of Computer Vision, 126(7):671-688, 2018.

[20] T. Marrin and R. Picard. The "conductor's jacket": A device for recording expressive musical gestures. In Proceedings of International Computer Music Conference, 1998.

[21] S. Mitra and T. Acharya. Gesture recognition: A survey. IEEE Transactions on Systems man and Cybernetics C, 37(3):311-324, 2007.

[22] S. Moccia, L. Migliorelli, V. Carnielli, and E. Frontoni. Preterm infants' pose estimation with spatio-temporal features. IEEE Transactions on Biomedical Engineering, 2019.

[23] F. R. Moore. The dysfunctions of midi. Computer music journal, 12(1):19-28, 1988.

[24] S. Y. Nikouei, Y. Chen, S. Song, and T. R. Faughnan. Kerman: A hybrid lightweight tracking algorithm to enable smart surveillance as an edge service. In 2019 16th IEEE Annual Consumer Communications \& Networking Conference (CCNC), pages 1-6. IEEE, 2019.

[25] W. Niu, J. Long, D. Han, and Y. Wang. Human activity detection and recognition for video surveillance. In Proc. of International Conference on Multimedia and Expo, pages 719-722, 2004.

[26] R. Ortale, E. Ritacco, N. Pelekis, R. Trasarti, G. Costa, F. Giannotti, G. Manco, C. Renso, and Y. Theodoridis. The daedalus framework: progressive querying and mining of movement data. In Proc. of International Conf. on Advances in Geographic Information Systems, pages 1-4, 2008.

[27] D. Overholt. The MATRIX: A novel controller for musical expression. In New Interfaces for Musical Expression, pages 38-41, 2001.

[28] L. Paolino, M. Romano, M. Sebillo, and G. Vitiello. Supporting the on-site emergency management through a visualisation technique for mobile devices. Journal of Location Based Services, 4(3-4):222-239, 2010.

[29] Y. Raaj, H. Idrees, G. Hidalgo, and Y. Sheikh. Efficient online multi-person 2D pose tracking with recurrent spatiotemporal affinity fields. In Proc. of IEEE Conference on Computer Vision and Pattern Recognition, pages 4620 4628, 2019.

[30] F. Remondino. Tracking of human movements in image space. Technical Report at IGP-ETH Zurich, 2001.

[31] F. Rumsey. MIDI systems and control. ButterworthHeinemann, 1994.

[32] A. Shehzed, A. Jalal, and K. Kim. Multi-person tracking in smart surveillance system for crowd counting and normal/abnormal events detection. In 2019 International Conference on Applied and Engineering Mathematics (ICAEM), pages 163-168. IEEE, 2019.

[33] M. Tattara and P. V. Aureli. The Room of One's Own. The Architecture of the (Private) Room. Black Square, 2017.

[34] H. Zhao, S. Wang, G. Zhou, and D. Zhang. Ultigesture: A wristband-based platform for continuous gesture control in healthcare. Smart Health, 11:45-65, 2019. 